\title{
A Fair Cooperative MAC Protocol in IEEE 802.11 WLAN
}

\author{
Seyed Davoud Mousavi ${ }^{1}$, Rasool Sadeghi ${ }^{2, *}$, Mohamadreza Karimi ${ }^{3}$, Erfan Karimian ${ }^{3}$ and \\ Mohammad Reza Soltan Aghaei ${ }^{1}$ \\ 1 Department of Computer, Isfahan (Khorasgan) Branch, Islamic Azad University, Isfahan 81595-158, Iran; \\ d_mousavi@tce.ir (S.D.M.); mersa6@yahoo.com (M.R.S.A.) \\ 2 Department of Electrical Engineering, Dolatabad Branch, Islamic Azad University, Isfahan 83418-57185, Iran \\ 3 Department of Computer Engineering, Dolatabad Branch, Islamic Azad University, Isfahan 83418-57185, Iran; \\ Mohamadrezakarimi64@gmail.com (M.K.); erfankarimian1366@gmail.com (E.K.) \\ * Correspondence: r.sadeghi@iauda.ac.ir; Tel.: +98-31-45-829-090
}

Received: 13 March 2018; Accepted: 3 April 2018; Published: 3 May 2018

\begin{abstract}
Cooperative communication techniques have recently enabled wireless technologies to overcome their challenges. The main objective of these techniques is to improve resource allocation. In this paper, we propose a new protocol in medium access control (MAC) of the IEEE 802.11 standard. In our new protocol, which is called Fair Cooperative MAC (FC-MAC), every relay node participates in cooperation proportionally to its provided cooperation gain. This technique improves network resource allocation by exploiting the potential capacity of all relay candidates. Simulation results demonstrate that the FC-MAC protocol presents better performance in terms of throughput, fairness, and network lifetime.
\end{abstract}

Keywords: cooperation; relay selection; fairness; lifetime

\section{Introduction}

Cooperative communications have emerged as a promising solution to some existing challenges for wireless networks. Cooperative communications provide performance improvement by exploiting a new paradigm beyond the conventional point-to-point and point-to-multipoint communication models. The broadcast nature of a wireless channel results in the overhearing of packets by the middle nodes between source and destination nodes. These middle nodes (relay or helper) can perform the forwarding when their participation leads to performance improvement. The capabilities of cooperative communications attract different wireless technologies and different OSI layers. The special features of IEEE 802.11 medium access control (MAC), integrated in cooperative schemes, have led the research community to provide a diversified set of solutions for different challenges, from rate adaptation techniques at the physical layer to service differentiation at the application layer and even cross-layer approaches [1-5].

To achieve the objectives of cooperative IEEE 802.11 MAC protocols, the relay selection mechanism is very important. The relay selection mechanism impacts on different aspects of network performance, such as spectrum efficiency, reliability, quality of service, energy efficiency, and fairness. In this paper, we propose a new cooperative protocol in IEEE 802.11 MAC which improves the network performance in terms of throughput, lifetime, and time fairness.

The rest of the paper is organized as follows: Section 2 discusses the state of the art of existing cooperative protocols. The proposed relay selection algorithm is described in Section 3. In Sections 4 and 5, we present performance analysis and simulation results of our proposed algorithm respectively. Section 6 concludes the paper and presents future directions for research. 


\section{Related Works}

The MAC protocols of the IEEE 802.11 standard have exploited cooperative techniques to provide improvements in spectrum efficiency and reliability efficiency. These techniques present two categories of cooperative MAC protocols in IEEE 802.11 networks. In the first category, the original cooperative MAC protocols in the IEEE 802.11 standard improve the network throughput by exploiting the optimum relay node providing the lowest delay, whereas in the second category, the cooperation mechanism improves energy efficiency. In this section, we present an overview of cooperative MAC protocols fitting these two categories.

CoopMAC [6] and rDCF [7] are the earliest protocols presented in the first category. In the CoopMAC protocol, each source node collects information on the transmission time by overhearing the control and data packet exchanged between its neighbors. This information is stored in a database called CoopTable. When the source node has a packet to send to an intended destination, the optimal relay node is selected from the CoopTable. Similarly, the rDCF protocol employs the minimum transmission time as the metric. However, the main difference between the CoopMAC and rDCF protocols is that CoopMAC obtains the decision information completely from overhearing whereas rDCF uses overhearing to obtain the primary information and then broadcasts this information to neighbors. The next protocol of this category is ADC-MAC [8], which employs the minimum transmission rate as a metric. In this protocol, a pure two-hop transmission is used when there is no available direct transmission and information on the lowest transmission time is broadcasted after the primary overhearing. Some protocols, such as RAMA [9] and EMR [10], exploit the metric of lowest transmission time with some modifications. In RAMA, the highest data rate is the metric for relay selection and the final relay is chosen through a random contention mechanism. The EMR selects the highest effective throughput as a metric and the winning relay is selected through a priority-based contention between relay candidates. The other protocols fitting this category can improve the performance of an IEEE 802.11 MAC protocol with some special techniques and features, such as frame aggregation in CoopMACA [11] and RRS-CMAC [12], concurrent transmissions in CCMAC [13], network coding in CODE [14] and EAP-CMAC [15], a cross-layer approach in MACR-CCT [16], interference reduction in CLS-MAC [17] and contention-based relay selection in RCD-MAC [18] and CRP-CMAC [19].

In the second category of cooperative MAC protocols, the cooperation condition and cooperation metric are dependent on power parameters, such as SNR and SINR. As an example, in opportunistic relaying (OR) [20], each relay node estimates the "instantaneous channel conditions" from overhearing control packets. Then, the relay which is able to provide the highest SNR will transmit a flag to the corresponding source and destination nodes. Another example of this category is a cooperative proposal referred to in the UTD-MAC protocol [21], in which power channel nformation among source, destination, and potential relays is evaluated by each source node. The relay node providing the maximum power ratio is the winning node. The operation of the UTD-MAC protocol is improved by other protocols with special features, such as coding schemes in CMAC [22] and CD-MAC [23], Automatic Repeat reQuest (ARQ)based cooperative MAC schemes in NCSW [24] and PRCSMA [25], and network coding in the NCCARQ-MAC protocol [26]. A summary of cooperative MAC protocols, their categories, and their particular techniques which provide performance improvement are presented in Table 1. The interested reader can refer to [1-4] for more information about the existing cooperative MAC protocols.

Nevertheless, the existing protocols select the relay based on the maximum available rates only and this leads to reuse of the same relay again and again. This would drain the energy of this relay and its outage and thus the protocol would lose a good cooperative partner. In our proposed protocol, we design an efficient scheme to provide a fair relay selection algorithm that can select the potential relays based on their cooperation gains. This would result in network lifetime maximization and fairness. 
Table 1. Summary of existing cooperative medium access control (MAC) protocols in the IEEE 802.11 standard.

\begin{tabular}{|c|c|c|}
\hline Category & Protocol & Technique \\
\hline \multirow{8}{*}{ Spectrum efficiency } & CoopMAC [6], rDCF [7] & $\begin{array}{l}\text { Replacing with two fast links instead of } \\
\text { a slow link }\end{array}$ \\
\hline & ADC-MAC [8] & Relaying when no direct link is available \\
\hline & $\begin{array}{l}\text { RAMA [9], EMR [10], RCD-MAC [18], } \\
\text { CRP-CMAC [19] }\end{array}$ & Contention-based relay selection \\
\hline & CoopMACA [11], RRS-CMAC [12] & Relaying + frame aggregation \\
\hline & CCMAC [13] & Relaying + concurrent transmissions \\
\hline & CODE [14] and EAP-CMAC [15] & Relaying + network coding \\
\hline & MACR-CCT [16] & Relaying + cross-layer approach \\
\hline & CLS-MAC [17] & Interference reduction \\
\hline \multirow{5}{*}{ Energy Efficiency } & OR [20] & $\begin{array}{l}\text { Selection of relay with the highest SNR } \\
+ \text { opportunistic relaying }\end{array}$ \\
\hline & UTD-MAC [21] & $\begin{array}{l}\text { Selection of relay node providing the } \\
\text { maximum power ratio }\end{array}$ \\
\hline & CMAC [22], CD-MAC [23] & Relaying + coding schemes \\
\hline & NCSW [24], PRCSMA [25] & Relaying + ARQ-based schemes \\
\hline & NCCARQ-MAC [26] & Relaying + network coding \\
\hline
\end{tabular}

\section{The Proposed FC-MAC Protocol}

In this section, we propose a new protocol which improves fairness and network lifetime. Therefore, we call this protocol Fair Cooperative MAC (FC-MAC). As indicated in Figure 1, node $R_{i}$ is a candidate relay between source $(S)$ and destination $(D)$. To find the potential relay node $\left(r_{i}\right)$ candidates, the FC-MAC protocol employs a metric called Cooperation Gain which can be calculated by

$$
C G_{i}=\left(\frac{R_{S r_{i}}^{-1}+R_{r_{i} D}^{-1}}{R_{S D}^{-1}}\right)^{-1}
$$

where, $R_{S r i}, R_{r i D}$, and $R_{S D}$ are the data rates of source-relay, relay-destination, and source-destination, respectively. These parameters are obtained from overhearing direct and indirect transmissions. A $C G_{i}$ value of more than 1 can provide for a database which maintains potential relays and their $C G_{i}$ parameters.

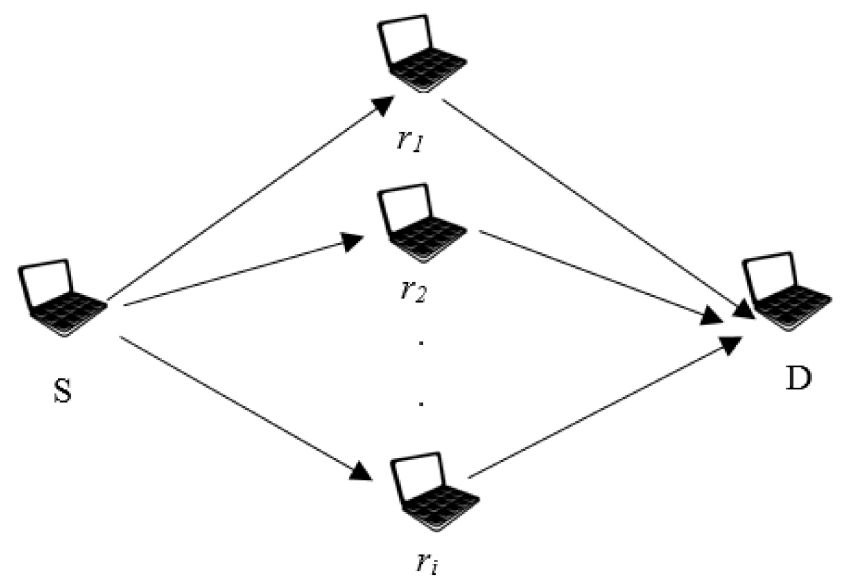

Figure 1. Example of cooperation with several relay nodes. $\mathrm{S}=$ source; $\mathrm{D}=$ destination. 
In order to provide fairness in the FC-MAC protocol, a new metric is defined to indicate the Cooperation Level (CL) of each relay node. For a potential relay $\left(r_{i}\right)$, the value of $C L_{i}$ is calculated by

$$
C L_{i}=\left[\frac{C G_{i}}{C G_{i-\min }}\right]
$$

where $C G_{i-\min }$ is the minimum value of $C G_{i}$ existing in the cooperation database and $[f()$.$] denotes the$ greatest integer function. By the $C L_{i}$ values, FC-MAC determines the cooperation level of all potential relay nodes. For a given destination, each source node has opportunities to select $k$ relay nodes from the set of $\left\{r_{1}, r_{2}, \ldots, r_{k}\right\}$ with cooperation levels of $\left\{C L_{1}, C L_{2}, \ldots, C L_{k}\right\}$. The number of packets which the source node allocates to each relay node to forward is equal to its $C L$ values. As a simple example, all possible scenarios of cooperation in IEEE $802.11 \mathrm{~b}$ are indicated in Table 2 when $R_{S D}=1 \mathrm{Mbps}$. According to this table, the ratio of relayed data packets in each scenario is determined. This ratio is 4 , 2 , and 1 for relays with a cooperation gain of 5.5, 3.67 (and 2.75), and 1.67 (and 1.47), respectively.

Table 2. FC-MAC scenarios in IEEE 802.11b ( $\left.\mathrm{R}_{\mathrm{SD}}=1 \mathrm{Mbps}\right)$.

\begin{tabular}{ccccccccc}
\hline $\boldsymbol{R}_{\text {Sri }}$ (Mbps) & $\mathbf{1 1}$ & $\mathbf{1 1}$ & $\mathbf{5 . 5}$ & $\mathbf{1 1}$ & $\mathbf{2}$ & $\mathbf{5 . 5}$ & $\mathbf{5 . 5}$ & $\mathbf{2}$ \\
\hline$R_{r i D}(\mathrm{Mbps})$ & 11 & 5.5 & 11 & 2 & 11 & 5.5 & 2 & 5.5 \\
$C G_{i}$ & 5.5 & 3.67 & 3.67 & 1.69 & 1.69 & 2.75 & 1.47 & 1.47 \\
$C L_{i}$ & 4 & 2 & 2 & 1 & 1 & 2 & 1 & 1 \\
\hline
\end{tabular}

The relay selection mechanism of the FC-MAC protocol is illustrated in Algorithm 1. Each time a source node has data packets to send, it allocates the data packets to each relay in proportion to its $C L_{i}$ parameter, and the parameters of CoopTable are updated as follows:

1. For a new added relay node, $C G_{i}$ and $C L_{i}$ are calculated by (1) and (2), respectively.

2. For an existing relay node in CoopTable (Table 3), its $C L_{i}$ is decreased by 1 if a transmission via the selected relay fails and it does not change for each successful transmission.

3. The relay node is removed from CoopTable when $C L_{i}=0$. However, the removed node can be added to CoopTable after a timeout.

4. The mechanism of sending control packets and data packets between the source, relay(s), and the destination is according to the Finite State Machine (FSM) of Figure 2. There exist extra states (e.g., WAITHTS, WAITDATA1, and WAITDATA2) which modify the Distributed Coordination Function (DCF) procedure. Moreover, two new data packets and three control packets are defined for exchanging between source, relay, and destination. The numbering 1 and 2 in Figure 2 refers to two phases of cooperation: $1=$ source to relay and $2=$ relay to destination.

5. After sending a modified RTS (called CoopRTS) packet via the source node containing the addresses of the destination and the intended relay node, a new control packet called Cooperative Helper-To-Send (CoopHTS) is sent by the relay node if it is ready to cooperate.

6. After the destination receives the modified CoopRTS and CoopHTS packets, it sends a CoopCTS packet to indicate to the source node to start data transmission.

7. By receiving the CoopCTS packet via the source node, the data packet is transmitted from source to relay (DATA1) and then forwarded from relay to destination (DATA2), respectively.

8. When the data packet of the second part (DATA2) is received successfully by the destination, it sends an $A C K$ packet to the source node.

9. During the cooperation phase, an expired timeout of control packets initiates the non-cooperation phase. In this case, the original RTS and CTS packets are employed to accomplish the transmission. 

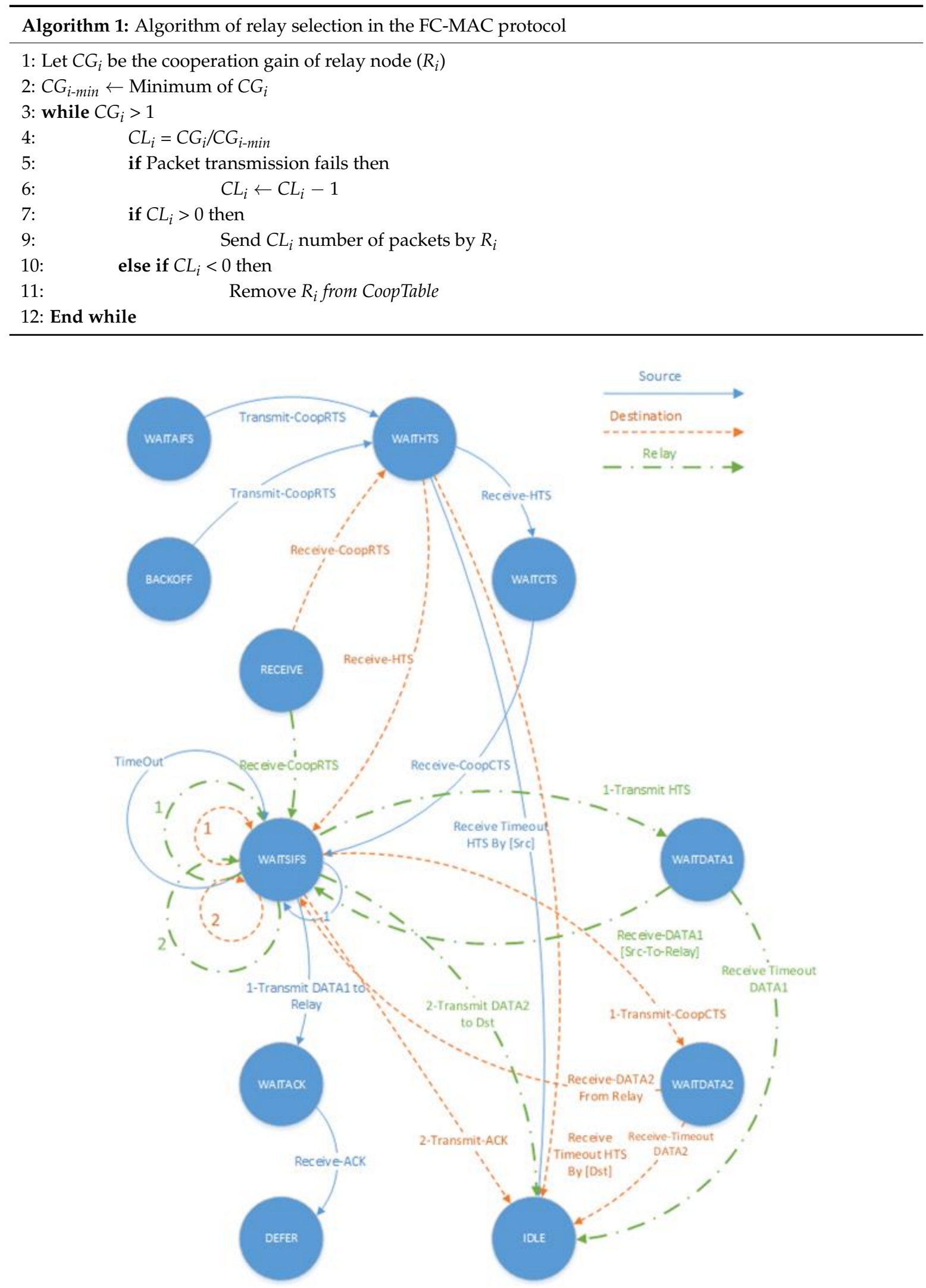

Figure 2. Finite State Machine of the FC-MAC protocol. 
Table 3. CoopTable of the FC-MAC protocol.

\begin{tabular}{cllllll}
\hline Relay ID & $R_{S D}$ & $R_{S r i}$ & $R_{r i D}$ & $C G_{i}$ & $C L_{i}$ & $\# A C K$ \\
\hline$R_{1}$ & & & & & & \\
$R_{2}$ & & & & & & \\
$\cdots$ & & & & & & \\
\hline
\end{tabular}

\section{Performance Analysis}

In this section, the performance of the FC-MAC protocol is analysed in terms of network throughput, time fairness, and network lifetime. We assume that the network is in saturation conditions and all nodes always have packets for transmission.

\subsection{Throughput}

According to the analysis in [26], A Markov model can describe the behaviour of a single node in the IEEE 802.11 standard. In the exponential back-off process, each node selects a random value in the contention window of [0, CWmin $]$ at the first transmission. If the packet transmission fails due to errors or a collision occurs, the length of the contention window is doubled, i.e., $C W=2^{i} C W_{\text {min }}$ and $i=0,1,2$, $\ldots, m$. After retransmission $m$ times, the packet will be dropped. When a network has $n$ nodes, the probability that there is at least one node transmitting a packet in a given time slot can be expressed as:

$$
P_{t r}=1-(1-\tau)^{n}
$$

where, $\tau$ denotes the transmission probability of a user in a particular time slot. For a no-collision scenario, the value of $\tau$ is obtained as [27]:

$$
\tau=\frac{2}{C W+1} .
$$

With the assumption of at least one node transmitting, the probability of a successful transmission can be calculated as follows:

$$
P_{S}=\frac{n \tau(1-\tau)^{n-1}}{P_{t r}} .
$$

The saturated throughput can be expressed by dividing the successfully transmitted payload information in a time slot, with the average length of a time slot, and we have

$$
S=\frac{P_{S} P_{t r} E[P]}{\left(1-P_{t r}\right) T_{i}+P_{t r} P_{S} T_{S}+P_{t r}\left(1-P_{S}\right) T_{C}}
$$

where $E[P], T_{i}, T_{s}$, and $T_{\mathcal{c}}$ denote the average packet length, the duration of an empty time slot, the successful transmission time, and the transmission time with a collision, respectively. In the basic access mode and RTS-CTS mode, we have

$$
\begin{gathered}
T_{S}^{b a s}=H+E\left[T_{D}\right]+T_{S I F S}+T_{A C K}+T_{D I F S} \\
T_{S}^{r t s}=H+E\left[T_{D}\right]+T_{R T S}+T_{C T S}+3 T_{S I F S}+T_{A C K}+T_{D I F S}
\end{gathered}
$$

and

$$
\begin{gathered}
T_{C}^{b a s}=H+E\left[T_{D}^{*}\right]+T_{D I F S} \\
T_{C}^{r t s}=T_{R T S}+T_{D I F S}
\end{gathered}
$$

where $H, E\left[T_{D}\right]$, and $E\left[T_{D}^{*}\right]$ denote the header of the physical layer and MAC layer, the average time to transmit a MAC frame (without MAC header), and the average time to transmit the longest packet payload involved in a collision, respectively. 
In the cooperation mode, we have

$$
T_{S}=P_{\text {Coop }} \cdot T_{S}^{\text {Coop }}+\left(1-P_{\text {Coop }}\right) \cdot T_{S}^{\text {Dir }}
$$

where $P_{\text {Coop }}, T_{S}{ }^{\text {Coop }}$, and $T_{S}$ Dir denote the cooperation probability in the network, the successful transmission time in cooperation mode, and the successful transmission time with no cooperation, respectively. The expression of $T_{S}{ }^{D i r}$ is the same as (7) and

$$
\begin{gathered}
T_{S}^{\text {Coop-bas }}=2 H+2 E\left[T_{D}\right]+2 T_{\text {SIFS }}+T_{A C K}+T_{D I F S} \\
T_{S}^{\text {Coop-rts }}=2 H+2 E\left[T_{D}\right]+T_{R T S}+T_{C T S}+T_{H T S}+5 T_{\text {SIFS }}+T_{A C K}+T_{D I F S} .
\end{gathered}
$$

\subsection{Network Lifetime}

If the average power of a node in the network is $\varepsilon$ and $E_{0}$ is the initial energy of the network, the network's lifetime can be expressed as

$$
L T=\frac{E_{0}}{\varepsilon} .
$$

\subsection{Time Fairness}

In most cooperative protocols, the tendency is to use a previous relay node that has provided a successful transmission. This leads to a much faster drain on the resources of successful relay nodes. Moreover, some potential relay nodes are excluded from cooperation to perform cooperation. To evaluate the time fairness of each node, we apply Jain's fairness index as in [28].

$$
\text { Jain's Index }=\frac{\left(\sum_{i=1}^{n} x_{i}\right)^{2}}{n \sum_{i=1}^{n} x_{i}^{2}}
$$

where $x i$ denotes the total data transmission time occupied by node $i$ among $n$ nodes.

\section{Simulation and Results}

To evaluate the FC-MAC protocol, we simulate a mobile ad-hoc network (MANET) with IEEE 802.11 DCF, the CoopMAC protocol [6], and the FC-MAC protocol using an event-driven simulator developed in the INET Framework of the OMNET++ [29]. Simulations were configured as default parameters of the IEEE $802.11 \mathrm{n}$ standard, which are summarized in Table 3. The simulation time is $100 \mathrm{~s}$ with a transmitted power of $100 \mathrm{~mW}$ and a packet size of 1024 bytes generated at a constant bit rate (CBR) of 2 Mbps UDP. Packets are transmitted in multi-rate mode for BER $<10^{-5}$ (Table 4 ). We used the Friis free space propagation model with a path loss exponent of 4 . Nodes are uniformly distributed over an area of $600 \mathrm{~m} \times 600 \mathrm{~m}$. In order to obtain established results, the curves presented were averaged over 50 runs.

Table 4. Simulation parameters of IEEE 802.11n.

\begin{tabular}{cccc}
\hline Parameter & Value & Parameter & Value \\
\hline RTS & $38.89 \mu \mathrm{s}$ & Slot time & $9 \mu \mathrm{s}$ \\
CTS and HTS & $32.23 \mu \mathrm{s}$ & SIFS & $10 \mu \mathrm{s}$ \\
CWmin & 15 slots & DIFS & $28 \mu \mathrm{s}$ \\
CWmax & 1023 slots & ACK & $32.23 \mu \mathrm{s}$ \\
\hline
\end{tabular}

The throughput of the FC-MAC protocol is depicted in Figure 3. Comparing the FC-MAC protocol with the IEEE 802.11 DCF and CoopMAC protocols demonstrates the performance improvement of the FC-MAC protocol. Both the CoopMAC and FC-MAC protocols have better performance than the DCF protocol. When the number of nodes increases, the spectrum efficiency provided by the FC-MAC 
protocol is greater than the CoopMAC protocol because the FC-MAC protocol exploits the capacity of spectrum efficiency provided by all existing relay nodes, whereas the CoopMAC protocol is limited to the best relay node which presents the lowest transmission delay.

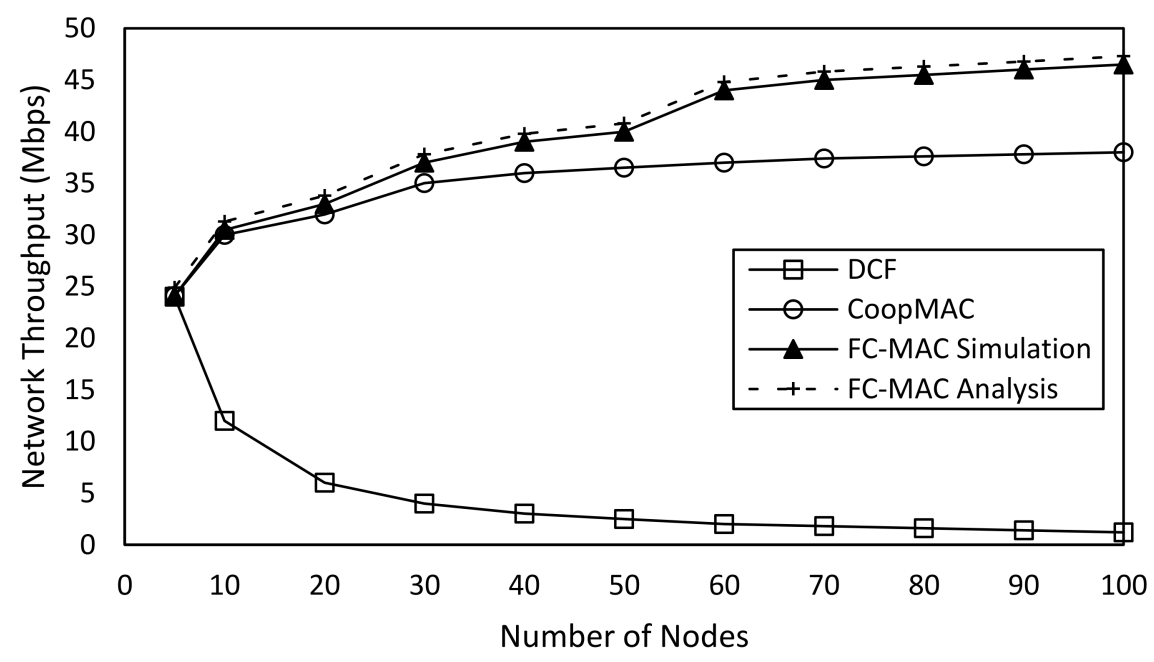

Figure 3. Network Throughput.

The network lifetime improvement of FC-MAC can be seen in Figure 4 when the number of nodes changes. As expected, the CoopMAC protocol achieves the shortest lifetime because the best relay nodes always forward packets for other nodes. The continual use of the best relay nodes in cooperation results in relay node outage and a reduction of network lifetime. The energy of each node in the DCF protocol is only consumed by direct transmission to the destination and there is no overhearing and forwarding due to cooperation. Therefore, the lifetime of the DCF protocol indicates better performance in comparison to the two other protocols. The relay selection scheme of the FC-MAC protocol provides balanced energy consumption. The FC-MAC protocol exploits the energy capacity of all nodes in the network. Thus, its lifetime outperforms the CoopMAC protocol while the forwarding mechanism leads to its degradation in comparison to the DCF protocol.

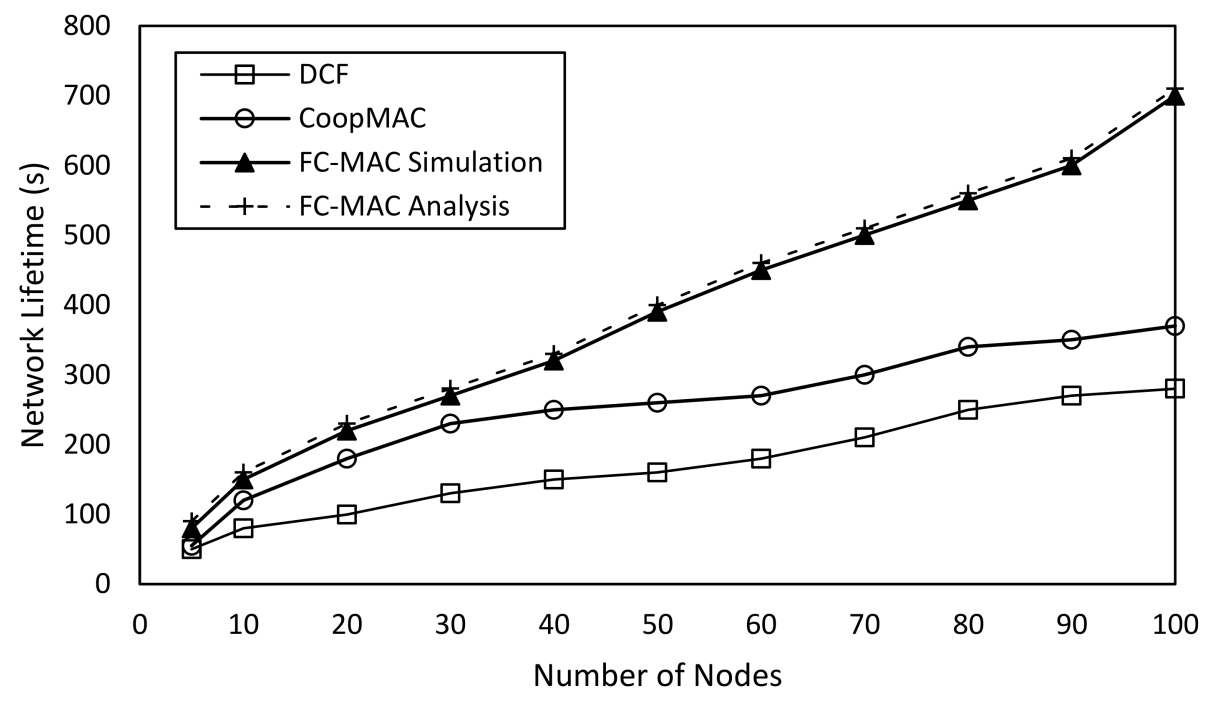

Figure 4. Network Lifetime.

Figure 5 indicates the time fairness of the FC-MAC, CoopMAC, and DCF protocols as the number of nodes increases. It can be observed that FC-MAC provides better time fairness than the other 
schemes. Since DCF and CoopMAC are not designed for time fairness, their fairness index is lower than that of FC-MAC. This is due to the fact that FC-MAC improves the time fairness based on high utilization of all existing relay nodes. Hence, all relay nodes with different cooperation gains have more chance to participate in cooperation and forwarding the packets.

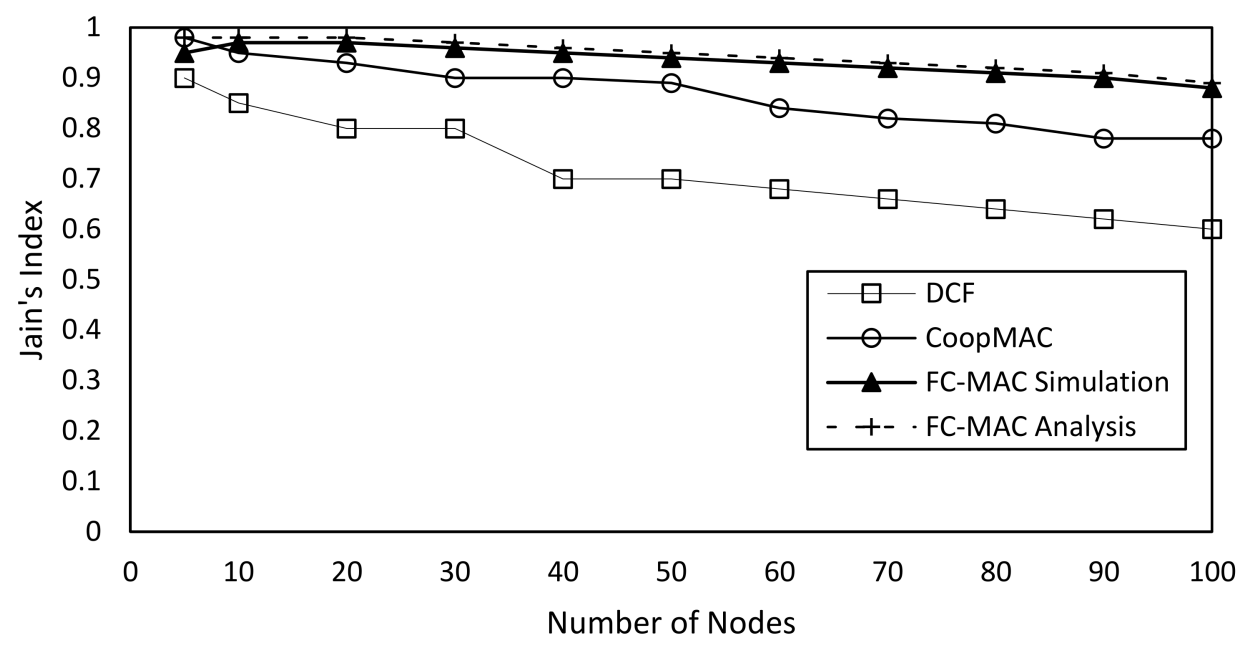

Figure 5. Time Fairness.

\section{Conclusions}

In this paper, we suggested a new cooperative MAC protocol in the IEEE 802.11 standard. The main goal of this protocol, called FC-MAC, is to improve time fairness and network lifetime. The improvement is obtained by employing all relay nodes with different cooperation gains. The number of forwarding packets by each relay node is proportional to its cooperation gain. This allocation is performed by the source node and after it evaluates the relay links and their reliability. The analysis and simulation results of the FC-MAC protocol demonstrate that the proposed relay selection scheme improves performance in terms of throughput, lifetime, and time fairness.

Author Contributions: The proposed protocol in this paper was carried out by Seyed Davoud Mousavi during his master thesis and under supervision of Rasool Sadeghi and Mohammad Reza Soltan Aghaei as an advisor. Seyed Davoud Mousavi wrote the initial draft of the paper from his master thesis while his supervisor provided the direction for their research activities and his advisor helped during the research activities and the paper writing.

Conflicts of Interest: The authors declare no conflict of interest.

\section{References}

1. Gomez-Cuba, F.; Asorey-Cacheda, R.; Gonzalez-Castano, F.J. A survey on cooperative diversity for wireless networks. IEEE Commun. Surv. Tutor. 2012, 14, 822-835. [CrossRef]

2. Ju, P.; Song, W.; Zhou, D. Survey on cooperative medium access control protocols. IET Commun. 2013, 7, 893-902. [CrossRef]

3. Zhuang, W.; Zhou, Y. A survey of cooperative MAC protocols for mobile communication networks. J. Internet Technol. 2013, 14, 541-559.

4. Silva, B.M.; Rodrigues, J.J.; Kumar, N.; Han, G. Cooperative strategies for challenged networks and applications: A survey. IEEE Syst. J. 2017, 11, 2749-2760. [CrossRef]

5. Sadeghi, R.; Barraca, J.P.; Aguiar, R.L. A Survey on Cooperative MAC Protocols in IEEE 802.11 Wireless Networks. Wirel. Person. Commun. 2017, 1-25. [CrossRef]

6. Liu, P.; Tao, Z.; Narayanan, S.; Korakis, T.; Panwar, S.S. CoopMAC: A cooperative MAC for wireless LANs. IEEE J. Sel. Areas Commun. 2007, 25, 340-354. [CrossRef]

7. Zhu, H.; Cao, G. rDCF: A relay-enabled medium access control protocol for wireless ad hoc networks. IEEE Trans. Mob. Comput. 2006, 5, 1201-1214. [CrossRef] 
8. Zhou, T.; Sharif, H.; Hempel, M.; Mahasukhon, P.; Wang, W.; Ma, T. A novel adaptive distributed cooperative relaying MAC protocol for vehicular networks. IEEE J. Sel. Areas Commun. 2011, 29, 72-82. [CrossRef]

9. Zou, S.; Li, B.; Wu, H.; Zhang, Q.; Zhu, W.; Cheng, S. A relay-aided media access (RAMA) protocol in multirate wireless networks. IEEE Trans. Veh. Technol. 2006, 55, 1657-1667. [CrossRef]

10. Pathmasuritharam, J.S.; Das, A.; Gupta, A.K. Efficient multi-rate relaying (EMR) MAC protocol for ad hoc networks. In Proceedings of the IEEE International Conference on Communications, Seoul, Korea, 16-20 May 2005; Volume 5, pp. 2947-2951.

11. Jibukumar, M.G.; Datta, R.; Biswas, P.K. CoopMACA: A cooperative MAC protocol using packet aggregation. Wirel. Netw. 2010, 16, 1865-1883. [CrossRef]

12. Liu, K.; Chang, X.; Liu, F.; Wang, X.; Vasilakos, A.V. A cooperative MAC protocol with rapid relay selection for wireless ad hoc networks. Comput. Netw. 2015, 91, 262-282. [CrossRef]

13. Hu, Z.; Tham, C.K. CCMAC: Coordinated cooperative MAC for wireless LANs. Comput. Netw. 2010, 54, 618-630. [CrossRef]

14. Tan, K.; Wan, Z.; Zhu, H.; Andrian, J. CODE: Cooperative medium access for multirate wireless ad hoc network. In Proceedings of the 2007 th Annual IEEE Communications Society Conference on Sensor, Mesh and Ad Hoc Communications and Networks, San Diego, CA, USA, 18-21 June 2007; pp. 1-10.

15. Sami, M.; Noordin, N.K.; Hashim, F.; Subramaniam, S.; Akbari-Moghanjoughi, A. An Energy-Aware Cross-Layer Cooperative MAC Protocol for Wireless Ad Hoc Networks. J. Netw. Comput. Appl. 2015, 58, 227-240. [CrossRef]

16. Wu, Q.; Zhou, X.; Ge, F. A cross-layer protocol for exploiting cooperative diversity in multi-hop wireless ad hoc networks. Wirel. Netw. 2016, 23, 1591-1610. [CrossRef]

17. Cui, H.; Li, J.; Li, Z.; Pan, D.; He, Y. Distributed Interference-Aware Cooperative Random Access in Multi-Hop Wireless Networks. IEEE Access 2016, 4, 4823-4828. [CrossRef]

18. Li, Y.; Liu, K.; Liu, F.; Xu, Z. A rapid cooperation-differentiated medium access control protocol with packet piggyback for multihop wireless networks. EURASIP J. Wirel. Commun. Netw. 2013, 2013, 1-15. [CrossRef]

19. Li, Y.; Liu, K.; Liu, F. CRP-CMAC: A Priority-Differentiated Cooperative MAC Protocol with Contention Resolution for Multihop Wireless Networks. TIIS 2013, 7, 2636-2656.

20. Cetin, B. Opportunistic relay protocol for IEEE 802.11 WLANs. Master's Thesis, Royal Institute of Technology, Stockholm, Sweden, 2006.

21. Agarwal, N.; Agarwal, N.; Kannan, L.N.; Tacca, M.; Fumagalli, A. IEEE 802.11b cooperative protocols: A performance study. In Proceedings of the IFIP/TX6 Networking Conference, Atlanta, GA, USA, 14-18 May 2007; pp. 415-426.

22. Chou, C.T.; Ghosh, M. Cooperative communication MAC (CMAC)-a new MAC protocol for next generation wireless LANs. In Proceedings of the 2005 International Conference on Wireless Networks, Communications and Mobile Computing, Maui, HI, USA, 13-16 June 2005; Volume 1, pp. 1-6.

23. Moh, S.; Yu, C.; Park, S.M.; Kim, H.N.; Park, J. CD-MAC: Cooperative diversity MAC for robust communication in wireless ad hoc networks. In Proceedings of the 2007 IEEE International Conference on Communications, Glasgow, UK, 24-28 June 2007; pp. 3636-3641.

24. Dianati, M.; Ling, X.; Naik, K.; Shen, X. A node-cooperative ARQ scheme for wireless ad hoc networks. IEEE Trans. Veh. Technol. 2006, 55, 1032-1044. [CrossRef]

25. Alonso-Zarate, J.; Kartsakli, E.; Verikoukis, C.; Alonso, L. Persistent RCSMA: A MAC protocol for adistributed cooperative ARQ scheme in wireless networks. EURASIP J. Adv. Signal Process. 2008, 2008. [CrossRef]

26. Antonopoulos, A.; Verikoukis, C.; Skianis, C.; Akan, O.B. Energy efficient network coding-based MAC for cooperative ARQ wireless networks. Ad Hoc Netw. 2013, 11, 190-200. [CrossRef]

27. Bianchi, G. Performance analysis of the IEEE 802.11 distributed coordination function. IEEE J. Sel. Areas Commun. 2000, 18, 535-547. [CrossRef]

28. Jain, R.; Durresi, A.; Babic, G. Throughput Fairness Index: An Explanation; ATM Forum/99-0045, February 1999; The Ohio State University: Columbus, OH, USA, 1999.

29. OMNeT++ Discrete Event Simulator. Available online: https://omnetpp.org/ (accessed on 1 March 2018).

(C) 2018 by the authors. Licensee MDPI, Basel, Switzerland. This article is an open access article distributed under the terms and conditions of the Creative Commons Attribution (CC BY) license (http:/ / creativecommons.org/licenses/by/4.0/). 